\title{
The Demographic Analyses of Undiagnosed and Misdiagnosed Cancer Cases via Autopsy Data
}

\author{
Aishvarya Godla ${ }^{1}$, Andrew Zloza ${ }^{\#}$, and Marco Rossi ${ }^{\#}$ \\ Hinsdale Central High School, Hinsdale, IL, USA \\ "Advisor
}

\section{$\underline{\text { ABSTRACT }}$}

Cancer is one of the most common causes of death. Recent increases in undiagnosed and misdiagnosed cancer cases at autopsies suggests that the presence of cancer is more prevalent in the general population than previously thought. Such cases may be different in certain populations with regard to key demographic characteristics (age, race, gender, etc.). Therefore, this study aimed to identify trends in these undiagnosed cases of different cancer types. The data suggest that cancers of the lung, gastrointestinal tract, and prostate gland were frequently undiagnosed/misdiagnosed, while cancers of the breast and brain are rarely missed. The data also show that the presence of undetected prostate cancer is likely not different, to a significant degree, in younger versus older age groups. This indicates that cancer possibly manifests early in human life but remains undetected for several decades. However, further research is required to confirm this hypothesis. The data suggests that the prevalence of undetected cancer is highest in AfricanAmericans compared to other races/ethnicities such as European and Asian. With regard to gender, the prevalence of undetected cancer appears to be higher in men than women. To conclusively validate these findings, further research is needed including concurrent and prospective analyses on live cases.

\section{Introduction}

Cancer is the second leading cause of death in the United States, with 1 in 6 people dying due to the disease (Roser et al., 2019). Additionally, cancer is an expensive as well as physically and mentally exhausting disease. It has emerged as a major public health issue because of the increased prevalence of cancer risk factors (Stewart et al., 2018).

The presence of cancer is more prevalent in the general, otherwise healthy populace than previously thought. Latest estimates from the organization Cancer Research UK indicate that 1 in 2 people is likely to develop cancer at some point in their lifetime (Jones et al., 2015). It is reasonable to suspect that increased prevalence leads to higher incidences of undiagnosed or misdiagnosed cases- both recorded and unrecorded. This may be an indication that cancer can manifest in people at early ages but remain undetected for longer than we think. Such undiagnosed and misdiagnosed cases may differ based on key demographic factors, specifically age, race, and gender. This study aims to investigate trends in the undiagnosed or misdiagnosed cases of different cancer types.

\section{Methods}

This study focused on collecting information from cancer autopsy cases found in previously conducted studies. The number of incorrectly diagnosed cases and three demographic characteristics of these cases- age, race, and gender, were collected. The inclusion of data was not limited to any one type of cancer. This study collected data from primary literature, reviews, and meta-analyses. The databases used in the process of data collection were EBSCO, PubMed, NCBI, and Springer Link. Keywords used were "cancer", "autopsy", "undiagnosed", and "misdiagnosed". Patients 
being screened for the included studies were each selected on a simple randomized approach, with specific studies selecting samples based on certain demographics (age, gender, race, etc.)

\section{General}

The studies by Burton et al., Sens et al., Bauer et al., and Subramony et al. collected and examined data from autopsies with ex-post cancer diagnosis. Neoplasms were classified as benign or malignant. Cases where cancer was found at autopsy but no clinical impressions of cancer were found before the autopsy were classified as unsuspected cancer. (Burton et al; Sens et al, Bauer et al; Subramony et al).

\section{Prostate Cancer}

The study by Bell et al. included autopsy studies of adult men (age $\geq 18$ years) who had no history of preexisting prostate cancer but included a systematic histological examination of the prostate gland. The study excluded studies that did not report the age of men, did not methodically examine the prostate microscopically, or were smaller than 100 men (Bell et al.). The study by Jahn used prostate cancer autopsy data from 40 studies. The study excluded data that included men with a clinical diagnosis of prostate cancer or did not stratify by age (Jahn et al., 2015).

\section{Breast Cancer}

The study by Thomas et al. included autopsy studies of women with no history of breast pathology, which included systematic histological examination of at least one breast and allowed the calculation of the prevalence of incidental breast cancer or precursor lesions (Thomas et al., 2017).

\section{Results}

\section{Characterization of Undiagnosed and Misdiagnosed Cancers:}

The study by de Pangher Manzini indicated that there was no clinically significant difference in the number of cancer cases in patients younger versus those older than 65 (de Pangher Manzini et al., 2010).

The data also suggest that undetected cancer prevalence (for any type) was highest among African-Americans. The study by Burton et al. stated that there was a 7:3 black to white ratio for the autopsies with malignant cancer (Burton et al., 1998). The study by Subramony stated that the highest number of cancers found in autopsies were among African-Americans- at 70\% (Subramony et al., 2003).

Undetected cancer prevalence (for any type) was higher among men than women. In the studies by Burton et al., Sens et al., and Bauer et al., the differences of cancer incidence between men and women were $26 \%, 25 \%$, and $22 \%$, respectively (Burton et al.; Sens et al.; Bauer et al.).

Frequently Undiagnosed/Misdiagnosed:

Lung cancer was consistently noted to be the most undiagnosed or misdiagnosed cancer in the observed literature. According to a study by Burton et al., 37\% of respiratory tract cancer cases were undiagnosed, making it the most frequently misdiagnosed. The study also states that the most common lung cancer histology at autopsy was adenocarcinoma (Burton et. al). Further studies attest to the notion that lung cancer is the most frequently misdiagnosed disease. Bauer et al demonstrated that the highest number of misdiagnoses fall under lung-bronchus cancer (Bauer et al., 1972). Lung carcinomas were the most prevalent malignant neoplasms only detected at autopsy in a study by Subramony. 
Another common disease site for undiagnosed and misdiagnosed cancers is the pancreas. The study by Sens et al. states that the incidence of unsuspected pancreatic malignant neoplasms was similar to the incidence of undiagnosed lung cancers (Sens et al., 2009). A study by Karwinski et al. corroborates the above findings- in that the unrecognized cancers that caused death were most often secondary to lung or pancreas (Karwinski et al., 1990).

The data suggest that following respiratory cancers and pancreatic cancers, the highest number of undiagnosed or misdiagnosed cases fall under the category of gastrointestinal cancer (GI) or prostate cancer. A study by Burton et al. states that $25 \%$ of GI cases were undiagnosed, just behind respiratory cancer cases. A study by Karwinski et al. corroborates the above findings in that gastric cancer ranks second among the unrecognized cancers investigated that caused death.

There are some discrepancies between the different studies. Some reports indicate higher incidence of GI cancer while others indicate a higher number of incorrectly diagnosed prostate cancers. A study by Subramony states that the number of prostate carcinomas detected ranks second on the list (with lung carcinomas ranking the first). The number of stomach carcinoids and colon carcinoma ranks sixth and seventh respectively on the list. A study by Sens et al. states that behind pancreas and lung cancer, kidney cancer has the highest number of unsuspected malignant cancers. Directly following kidney cancer is prostate cancer. The study reported no unsuspected malignant neoplasms found in cases of stomach cancer. Finally, a study by Bauer et al. stated that lung cancer and colon cancer have the highest incorrect diagnosis rate and following those is prostate cancer and finally stomach cancer (Bauer et. al).

It is commonly known that the prevalence of any type of cancer increases with age. A study by Bell et al. shows that prostate cancer prevalence increases with each decade, and this relationship is statistically significant as the odds ratio calculated for cases used without the Gleason score was 1.7, while for cases with a Gleason score was 2.0. The study also calculated that the mean cancer prevalence for people of age 30 and under was lower (5\%), compared to 59\% for ages 79 and above (Bell et al.). A study by Bubendorf et al. states that a strong relationship is present between patient age and prevalence of undetected prostate cancer: $0.2 \%$ in ages $40-49$, and $16.1 \%$ in ages 80 and above (Bubendorf et al.). A study by Jahn et al. agrees with the above findings and states that a high prevalence of undetected prostate cancer is present in ages 60-69. However, it also notes a high prevalence of undetected prostate cancer in ages 30-40 between 23 and 35\% (Jahn et. al). These findings do not support the trend that cancer prevalence increases with age, as suggested by other numerous studies.

In terms of race/ethnicity, undetected prostate cancer prevalence was found to be highest among AfricanAmericans. A study by Bell et al. states that prostate cancer prevalence is lowest in Asian men, intermediate in European men, and highest in African men. However, it was not reported whether these findings were statistically significant. (Bell et. al). A study by Jahn et al. corroborates the study by Bell et al. for every decade of age- Asian men had the lowest prevalence of cancer, white men had an intermediate prevalence of cancer, and African men had the highest prevalence of cancer (Jahn et al.).

\section{Rarely Undiagnosed and Misdiagnosed:}

The data show that breast cancer is rarely undiagnosed. Studies by Sens et al., Bauer et al., Burton et al., and Thomas et al. all noted that the incidence of undiagnosed breast cancer at autopsy consistently ranked the lowest out of all other cancers. The median rate of undiagnosed invasive breast cancer was shown to be $1.3 \%$ (Thomas et al.). Studies by Burton et al. and Bauer et al. also suggest a very low incidence of undiagnosed brain cancer cases.

\section{Discussion}

The cancers that are frequently undiagnosed or misdiagnosed are lung, pancreatic, prostate and GI, while the cancers that are rarely missed are breast and brain. Prostate cancer has been thoroughly researched and some studies suggest 
that the prevalence of undetected cancer is high in both older and younger men. There is not enough research to determine whether the incidence of other undiagnosed or misdiagnosed cancers changes with age.

One possible explanation for the high incidence of undiagnosed lung cancer in the studies reviewed is the lack of CT lung screening until recently. Similarly, there is not adequate screening to detect pancreatic and gastric cancers.

With the results described, it appears that the factors of race/ethnicity contribute to the prevalence of most undiagnosed and misdiagnosed cases of prostate cancer. In studies by Bell et al. and Jahn et al., the highest group of people with undetected prostate cancer were African-American. However, age does not appear to play a major factor in the study by Jahn et al. This may be an indication that cancer can manifest in people at early ages but remain undetected for longer than we think.

Amongst the studies reviewed, the prevalence of undetected cancer was higher in men than in women. This may be explained by the fact that prostate cancer affects only men. Prostate cancer is also extremely common, and 1 in 9 men will be diagnosed with prostate cancer during their lifetime (American Cancer Society).

The lack of representation of different races/ethnicities in the data is a major issue. Among all of the studies the only races/ethnicities consistently analyzed were European and African-American. In future studies, it would be more accurate and beneficial to collect data from autopsies from a wide range of races/ethnicities to ensure that the sampling is representative.

\section{Conclusion}

With cancer being so common, it is extremely crucial to understand the characteristics and attributes of undiagnosed and misdiagnosed cases. To accomplish this, cancer autopsy data (not limited to any one type of cancer) was reviewed from previously conducted meta-analyses as well as the primary literature and review articles. From the literature examined, lung cancer, pancreatic cancer, gastrointestinal cancer, and prostate cancer were frequently undiagnosed or misdiagnosed, while breast cancer and brain cancer were rarely undiagnosed or misdiagnosed.

While the data presented shows that the prevalence of undetected prostate cancer is equally high in both young and old age groups, more research is needed to fully confirm this finding. The prevalence of undetected prostate cancer was highest in African-American men, compared to other races/ethnicities. While this may be true, it is not representative of the general population as other races/ethnicities need to be analyzed more comprehensively.

Finally, the prevalence of undetected cancer in general was higher in men than women. This concurs with the trend that certain cancers that only affect men (prostate cancer) were more often undiagnosed or misdiagnosed than cancers that mainly affect women (breast cancer).

Looking forward, further studies comparing the rate of these cases in different demographic groups (age, race, and gender) for all types of cancers would greatly benefit the analysis of undiagnosed and misdiagnosed cases of cancer, and may lead to changes in public health recommendations (e.g., clinical monitoring at earlier ages or via more advanced technologies).

\section{Acknowledgments}

I thank Dr. Andrew Zloza and Mr. Marco Rossi of Rush University for guiding me through this project and revising my scientific paper.

\section{References}

AM;, S. M. (n.d.). Unexpected neoplasia in autopsies: Potential implications for tissue and organ safety. Retrieved from https://pubmed.ncbi.nlm.nih.gov/19961246/ 
Bell, K. J., Del Mar, C., Wright, G., Dickinson, J., \& Glasziou, P. (2015, October 01). Prevalence of incidental prostate cancer: A systematic review of autopsy studies. Retrieved from https:/www.ncbi.nlm.nih.gov/pmc/articles/PMC4682465/

Bubendorf, L., Schöpfer, A., Wagner, U., Sauter, G., Moch, H., Willi, N., . . Mihatsch, M. J. (2005, March 17). Metastatic patterns of prostate cancer: An autopsy study of 1,589 patients. Retrieved from https:/www.sciencedirect.com/science/article/abs/pii/S0046817700800350?via=ihub Elizabeth C. Burton, M. (1998, October 14). Autopsy Diagnoses of Malignant Neoplasms. Retrieved from https://jamanetwork.com/journals/jama/fullarticle/188050

Frederick W. Bauer, M. (1972, September 25). An Autopsy Study of Cancer Patients. Retrieved from https://jamanetwork.com/journals/jama/article-abstract/344534

HG. Welch, W. B., A. Bleyer, H. W., LJ. Esserman, I. T., MG. Marmot, D. A., Barratt, A., J. Carter, R. C., . . NA. de Glas, M. K. (1970, January 01). Prevalence of incidental breast cancer and precursor lesions in autopsy studies: A systematic review and meta-analysis. Retrieved from https://bmccancer.biomedcentral.com/articles/10.1186/s12885017-3808-1

Jahn, J. L., Giovannucci, E. L., \& Stampfer, M. J. (2015, January 08). The high prevalence of undiagnosed prostate cancer at autopsy: Implications for epidemiology and treatment of prostate cancer in the Prostate-specific Antigenera. Retrieved from https://onlinelibrary.wiley.com/doi/full/10.1002/ijc.29408

Karwinski, B., Svendsen, E., \& Hartveit, F. (2009, August 15). Clinically undiagnosed malignant tumours found at autopsy. Retrieved from https:/onlinelibrary.wiley.com/doi/abs/10.1111/j.1699-0463.1990.tb01062.x

PMC, E. (n.d.). Retrieved from http://europepmc.org/article/med/7890278

Shibahara, Y., Sugawara, Y., Miki, Y., Hata, S., Takahashi, H., Nakamura, Y., . . Sasano, H. (2016, November 22). Analysis of multiple primary cancer autopsy cases associated with breast cancer: 2002-2010. Retrieved from https://onlinelibrary.wiley.com/doi/abs/10.1111/pin.12484

Subramony, C. (2003, October 01). Clinically Undiagnosed Non-Neoplastic and Neoplastic Diseases Found on Postmortem Examination. Retrieved from https://academic.oup.com/labmed/article/34/10/743/2657270 